\title{
Properties determined by the Ihara zeta function of a Graph
}

\author{
Yaim Cooper * \\ Department of Mathematics \\ Princeton, NJ, USA \\ yaim@math.princeton.edu
}

Submitted: Nov 1, 2008; Accepted: Jun 22, 2009; Published: Jul 9, 2009

Mathematics Subject Classification: 05 C99

\begin{abstract}
In this paper, we show how to determine several properties of a finite graph $G$ from its Ihara zeta function $Z_{G}(u)$. If $G$ is connected and has minimal degree at least 2 , we show how to calculate the number of vertices of $G$. To do so we use a result of Bass, and in the case that $G$ is nonbipartite, we give an elementary proof of Bass' result. We further show how to determine whether $G$ is regular, and if so, its regularity and spectrum. On the other hand, we extend work of Czarneski to give several infinite families of pairs of non-isomorphic non-regular graphs with the same Ihara zeta function. These examples demonstrate that several properties of graphs, including vertex and component numbers, are not determined by the Ihara zeta function. We end with Hashimoto's edge matrix T. We show that any graph $G$ with no isolated vertices can be recovered from its $T$ matrix. Since graphs with the same Ihara zeta function are exactly those with isospectral $\mathrm{T}$ matrices, this relates again to the question of what information about $G$ can be recovered from its Ihara zeta function.
\end{abstract}

\section{Introduction and Statement of Results}

In this paper we study properties of graphs which are determined by the Ihara zeta function. Here, we allow multiple edges and loops in graphs. The Ihara zeta function, defined below, was introduced by Ihara in 1966 [4], and in its present form associates to each finite graph a zeta function. In the remainder of this paper, we will generally refer to this function simply as the zeta function. It has long been known that for any graph $G$ whose minimal degree is at least 2, the number of edges of $G$ can be computed from $Z_{G}(u)$ by considering the degree of the polynomial $Z_{G}(u)^{-1}$ [1]. It is natural to

\footnotetext{
*Supported by NSF grant DMS-0353722 and Louisiana Board of Regents Enhancement grant LEQSF (2005-2007)-ENH-TR-17.
} 
ask then whether the Ihara zeta function determines other properties such as the vertex number, component number, or regularity of an arbitrary graph $G$. In fact, examples from Czarneski [2] demonstrate that in general the Ihara zeta function determines none of these quantities.

On the other hand, when we restrict to graphs with a single connected component, the story becomes much nicer. A natural framework in which to study the zeta function involves regarding $G$ as the quotient of an infinite tree $X$ by an automorphism group $\Gamma$. This quotient always results in a connected graph, so it is natural to expect that the Ihara zeta function has nice properties on connected graphs which it loses when it is extended to multiple component graphs. An important result in this direction is the following.

Theorem 1. Let $G$ be a connected graph with minimal degree at least 2. Then it can be determined from the zeta function whether $G$ is

i) not bipartite,

ii) bipartite but not cyclic, or

iii) bipartite and cyclic,

and in these cases the vertex number $n$ of $G$ is given by

i) $n=e-\delta$

ii) $n=e-\delta+1$

iii) $n=e-\delta+2$

where $\delta$ is the number of times $1-u^{2}$ divides $Z_{G}(u)^{-1}$ and $e$ is the number of edges of $G$.

We can apply this result to study regular graphs and their spectra. The spectrum of a graph refers to the eigenvalues of its adjacency matrix.

Theorem 2. If $G$ is a connected graph with minimal degree at least 2, then from the zeta function $Z_{G}(u)$ it can be determined whether $G$ is regular. Moreover, if $G$ is regular, the regularity and spectrum of $G$ can be computed from $Z_{G}(u)$.

Aubi Mellein proved that if $G$ and $G^{\prime}$ are both known to be regular, then their zeta functions are equal if and only if they have the same spectrum [5]. Theorem 2 is a strengthening of this result.

The proof of the previous two theorems rely on a theorem of Bass' in [1]. In the case that $G$ is nonbipartite, we give an elementary proof of the necessary theorem.

Theorem 3. Let $G$ be a connected nonbipartite graph with minimal degree at least 2 . Then $\left(1-u^{2}\right)$ does not divide $\operatorname{det}\left(I-A u+Q u^{2}\right)$.

In the previous theorem, $A$ denotes the adjacency matrix and $Q$ the degree minus one matrix, both defined in the next section.

Finally, we explore the relation of $G$ and $Z_{G}(u)$ to the edge adjacency matrix $T$, which was introduced by Hashimoto [3]. For a graph with minimal degree at least $2, Z_{G}(u)$ is closely related to the spectrum of the T matrix. Specifically, if $z_{1} \ldots z_{2 e}$ are the roots of $Z_{G}(u)^{-1}$, then $z_{1}^{-1} \ldots z_{2 e}^{-1}$ are the eigenvalues of $T$. Therefore, the question of when two graphs have the same zeta function is equivalent to the question of when two graphs are isospectral with respect to the T matrix. This is analogous to the classic question of when 
are two graphs isospectral with respect to the adjacency matrix. The following theorem is therefore interesting: it shows that, just as a graph $G$ can be recovered from its adjacency matrix, $G$ can also be recovered from its $\mathrm{T}$ matrix.

Theorem 4. For a graph $G$ with no vertices of degree 0, $G$ can be recovered from the edge adjacency operator $T$.

In the following section, we establish the necessary definitions and preliminaries for the remainder of the paper. Then in Section 3 we work with vertex number and regularity, giving proofs for Theorem 1 and Theorem 2. In Section 4, we give an elementary proof of Theorem 3. In Section 5 we give examples of infinite families of pairs of non-isomorphic graphs with the same zeta function, which show that $Z_{G}(u)$ does not in general determine $G$, and that if $G$ is not required to be connected, $Z_{G}(u)$ does not even determine the vertex number or component number of $G$. Finally in Section 6 , we consider the edge adjacency matrix $T$ and prove Theorem 4 .

\section{Preliminaries}

The zeta function of a finite graph is defined analogously to the zeta function of an algebraic variety over a finite field.

Definition. A prime walk in a finite graph $G$ is a class of primitive closed backtrackless tailless walks in $G$, with $w \sim w^{\prime}$ if they are related by a cyclic permutation of vertices.

Here, a closed walk is one which begins and ends at the same vertex, a primitive closed walk is one which is not the power of any other, a backtrackless walk is one in which no edge is traversed and then immediately backtracked upon, and a tailless walk is one which is backtrackless under any cyclic permutation of vertices.

Definition. The degree or length of a prime walk $p$ is defined as the length of the path w, for any path $w$ that is a representative of the class $p$.

The length of a path is the number of edges the path passes through, counting multiplicity. That is, a single edge contributes as many times as the path passes through it. We now give the definiton of the zeta function of a finite graph $G$.

Definition. The Ihara zeta function $Z_{G}(u)$ of a graph $G$ is defined by

$$
Z_{G}(u)=\prod_{\text {prime walks } p \text { of } G \text { of nonzero length }}\left(1-u^{d_{p}}\right)^{-1}
$$

where $d_{p}$ is the length of the prime walk $p$.

Note that $Z_{G}(u)$ converges for small $|u|$.

In this paper, we allow multiple edges in a graph. By an md2 graph, we mean a graph $G$ with minimum degree at least 2. Equivalently $G$ is md2 if it contains no vertices of 
degree 1 or 0 . This is a natural class of graphs to consider with respect to the zeta function because adding vertices of degree 1 or 0 does not change $Z_{G}(u)$. The zeta function of $G$ depends only on the nontrivial prime walks in $G$ and adding a vertex of degree 0 adds no paths of nonzero length, so does not change $Z_{G}(u)$. Similarly, adding a vertex of degree 1 can only add paths of zero length or new paths with tails, and again these don't give rise to additional nontrivial prime walks.

Bass showed that if $G$ has minimal degree at least $2, Z_{G}(u)$ is the inverse of a polynomial [1].

$$
Z_{G}(u)=\frac{1}{\left(1-u^{2}\right)^{e-n} \operatorname{det}\left(I-A u+Q u^{2}\right)},
$$

where $A$ is the adjacency matrix of $G$ and $Q=D-I$ where $D$ is the diagonal matrix with $D_{i i}$ equal to the degree of vertex i.

Hashimoto gave another formula [3], valid when $G$ has minimal degree at least 2:

$$
Z_{G}(u)=\frac{1}{\operatorname{det}(I-T u)},
$$

where $T$ is the edge adjacency matrix of $G$, defined as follows.

First construct a digraph $\vec{G}$ on $n$ vertices by replacing each edge of $G$ with two oppositely oriented diedges $d$ and $\bar{d}$. If a diedge $d$ points from vertex $i$ to vertex $j$, then vertex $i$ is called the tail of $d$, and vertex $j$ is called the head of $d$.

Label the $2 e$ diedges of $\vec{G} d_{1} \ldots d_{2 e}$, with $d_{e+i}=\bar{d}_{i}, i=1,2 \ldots e$. A diedge $d_{i}$ is said to flow into a diedge $d_{j}$ if head $\left(d_{i}\right)=\operatorname{tail}\left(d_{j}\right)$ and $d_{i} \neq \bar{d}_{j}$.

Definition. The T matrix is defined by

$$
t_{i j}=\left\{\begin{array}{l}
1 \text { if } d_{i} \text { flows into } d_{j} \\
0 \text { otherwise }
\end{array}\right.
$$

Note that taking powers of $T$ generates the backtrackless, tailless walks through $G$.

\section{Computing invariants of $G$ from $Z_{G}(u)$}

\section{$3.1 \quad$ Vertex number}

It has long been known that the number of edges of any graph with minimal degree 2 can be computed from its zeta function [1]. This is easily seen from Bass' formula for the zeta function.

Lemma 5. If $G$ is md2, then the number of edges e of $G$ is given by $e=\frac{1}{2} \operatorname{deg}\left(Z_{G}(u)^{-1}\right)$

Proof. Because $G$ is $\operatorname{md} 2, \operatorname{det}(Q) \neq 0$. By equation $(2)$, the degree of $Z_{G}(u)^{-1}$ is $2 n-$ $2 n+2 e=2 e$.

We aim to show that vertex number and regularity are also determined by the zeta function. The result for vertex numbers will follow from the next lemma and theorem. 
Lemma 6. $Z_{G}(u)$ determines i) whether $G$ is bipartite and ii) whether $G$ is cyclic.

The following theorem was proved by Bass [1].

Theorem 7 (Bass). Suppose that $G$ is a connected md2 graph. Let $d$ be the number of times $1-u^{2}$ divides $\operatorname{det}\left(I-A u+Q u^{2}\right)$. Then

$$
d=\left\{\begin{array}{l}
0 \text { if } G \text { is nonbipartite } \\
1 \text { if } G \text { is bipartite but not a } 2 n \text { cycle } \\
2 \text { if } G \text { is a } 2 n \text { cycle. }
\end{array}\right.
$$

Now we combine Lemma 6 and Theorem 7 to prove Theorem 1 as follows:

Proof of Theorem 1. Recall Bass' formula for the zeta function of $G$

$$
Z_{G}(u)^{-1}=\left(1-u^{2}\right)^{e-n} \operatorname{det}\left(I-A u+Q u^{2}\right) .
$$

This formula shows that

$$
n=e-\delta+d
$$

where $\delta$ is the number of times $1-u^{2}$ divides $Z_{G}(u)^{-1}$ and $d$ is the number of times $1-u^{2}$ divides $\operatorname{det}\left(I-A u+Q u^{2}\right)$. By Lemma 6 , whether $G$ is bipartite or not and whether it is cyclic or not can be determined from $Z_{G}(u)$. Then Theorem 7 can be applied to obtain $d$. Equation (4) then becomes the formula given in the theorem statement.

Now we give a proof of Lemma 6 :

Proof. i) Whether $G$ is bipartite can be determined from $Z_{G}(u)$. To begin with, a graph $G$ is nonbipartite if and only if it contains a prime walk of odd degree.

$\Rightarrow G$ is nonbipartite, so $G$ contains an odd cycle $C$. $C$ then represents a prime walk of odd degree.

$\Leftarrow$ If $G$ is bipartite, then every closed walk has even degree, and hence every prime walk has even degree.

Next, $G$ does contain a prime walk of odd degree $\Leftrightarrow$ an odd power of u appears in the power series $Z_{G}(u)$.

To see this, consider the Euler product form of the Zeta function.

$$
\begin{aligned}
Z_{G}(u) & =\prod_{\text {prime walks p of } \mathrm{G} \text { of nonzero length }}\left(1-u^{\operatorname{deg}(p)}\right)^{-1} \\
& =\prod_{\text {prime walks p of nonzero length }}\left(1+u^{\operatorname{deg}(p)}+u^{2 \operatorname{deg}(p)}+u^{3 \operatorname{deg}(p)} \ldots\right) .
\end{aligned}
$$

Hence $G$ is not bipartite if and only if an odd power of $u$ appears in the power series $Z_{G}(u)$. Luckily, we know $Z_{G}(u)^{-1}$ is a polynomial, so it suffices to check whether any odd power of $u$ appears in $Z_{G}(u)^{-1}$.

ii) We can also determine whether $G$ is cyclic from $Z_{G}(u)$. In particular, $G$ is an n-cycle if and only if $Z_{G}(u)=\left(1+u^{n}+u^{2 n} \ldots\right)^{2}$. 
$\Rightarrow$ By direct computation.

$\Leftarrow$ If $Z_{G}(u)=\left(1+u^{n}+u^{2 n} \ldots\right)^{2}$, then $G$ has exactly two prime walks, each of length $n$. This implies that $G$ must be either an n-cycle or a linear graph of length n. (A linear graph of length $\mathrm{n}$ is a graph consisting of $\mathrm{n}+1$ vertices $v_{1} \ldots v_{n+1}$, and $\mathrm{n}$ edges joining $v_{1}$ and $v_{2}, v_{2}$ and $v_{3}, . . v_{n}$ and $v_{n+1}$. Such a graph can be drawn as a line segment divided into n edges, hence the name.) Then the assumption that $G$ is md2 forces $G$ to be an n-cycle.

\subsection{Regularity}

We are now ready to show that the regularity of $G$ can be determined from $Z_{G}(u)$.

Proof of Theorem 2. We can deduce results on regularity by comparing the trace and determinant of the degree matrix $Q$. The important fact is that both quantities can be obtained from $Z_{G}(u)$ now that $n$ and $e$ can be.

First, we recover $\operatorname{det}\left(I-A u+Q u^{2}\right)$ by

$$
\operatorname{det}\left(I-A u+Q u^{2}\right)=\frac{\left(1-u^{2}\right)^{n-e}}{Z_{G}(u)},
$$

as the quantities on the right can all be recovered from $Z_{G}(u)$. Because $G$ is md2, $\operatorname{det}(Q) \neq 0$ so $\operatorname{det}(Q)$ is the leading coefficient of $\operatorname{det}\left(I-A u+Q u^{2}\right)$ and we can recover $\operatorname{det}(Q)$. On the other hand, $\operatorname{tr}(Q)=2 e-n$ which now can also be computed from $Z_{G}(u)$.

A graph $G$ is regular if and only if $Q$ is a multiple of $I$ which by the inequality of arithmetic and geometric means occurs if and only if

$$
\operatorname{det}(Q)=\left(\frac{\operatorname{tr}(Q)}{n}\right)^{n} .
$$

In this case, the regularity of $G$ is $\frac{\operatorname{tr}(Q)}{n}+1$.

Hence $Z_{G}(u)$ determines whether $G$ is regular and if so its regularity. Now we show that if $G$ is regular, its spectrum can be computed from the zeta function.

The adjacency matrix A is always a real symmetric matrix, hence always diagonalizeable. On the other hand, if $G$ is regular then both $Q$ and $I$ are scalar matrices, so all three matrices can be simultaneously diagonalized and

$$
\operatorname{det}\left(I-A u+Q u^{2}\right)=\prod_{i}\left(q u^{2}-a_{i} u+1\right),
$$

where $q$ is one less than the regularity of $G$ and $a_{i}$ are the eigenvalues of $A$.

On the other hand, equation (5) showed that $\operatorname{det}\left(I-A u+Q u^{2}\right)$ can be recovered from $Z_{G}(u)$. Factoring this polynomial into linear terms and then pairing terms $c_{i} u-1, c_{j} u-1$ such that $c_{i} c_{j}=q$ recovers the eigenvalues of $A: a_{i}=c_{i}+c_{j}$.

Conversely, requiring only that $G$ be a regular md2 graph, $Z_{G}(u)$ can be recovered from the spectrum of $G$. Suppose the spectrum of $G$ is $\lambda_{1} \ldots \lambda_{n}$. Recall that the spectrum of 
$G$ is defined to be the spectrum of the adjacency matrix $A$. Hence from $\lambda_{1} \ldots \lambda_{n}$ we deduce that $A$ is conjugate to the diagonal matrix $L$ with $\lambda_{1} \ldots \lambda_{n}$ on its diagonal. Equivalently, $A=P L P^{-1}$ for an invertible matrix $P$. We also conclude that the number of vertices of $G$ is $\mathrm{n}$. It is well known that the regularity of $G, q+1$, is equal to the largest element of the spectrum of $G$. The number of edges $e$ is then $\frac{1}{2} n(q+1)$. Hence we have recovered both the matrix $Q=q I$, where I is the identity matrix, and the number of edges $e$. Finally because $I$ and $Q$ are scalar matrices, $\operatorname{det}\left(I-A u+Q u^{2}\right)=\operatorname{det}\left(P\left(I-A u+Q u^{2}\right) P^{-1}\right)=$ $\operatorname{det}\left(I-L u+Q u^{2}\right)$, which we can compute as we have recovered the matries $I, L$, and $Q$ already. Now $Z_{G}(u)$ can be computed as $\left[\left(1-u^{2}\right)^{e-n} \operatorname{det}\left(I-A u+Q u^{2}\right)\right]^{-1}$.

\section{An elementary proof in the case that $G$ is a non- bipartite connected md2 graph}

In the case that $G$ was nonbipartite, Theorem 7 was key to computing the quantity $n-e$, the number of vertices minus the number of edges, from $Z_{G}(u)$. In this section we give an elementary proof of that theorem in the case that $G$ is nonbipartite - that is, we prove Theorem 3, which states that in this case $\left(1-u^{2}\right) \operatorname{does} n o t \operatorname{divide} \operatorname{det}\left(I-A u+Q u^{2}\right)$. We do so by showing that $\operatorname{det}\left(I-A u+Q u^{2}\right)$ evaluated at $u=-1$ is nonzero. In particular, we show that $\operatorname{det}(I+A+Q)>0$. To begin, we introduce the auxilliary matrix

$$
Y=\left(\begin{array}{cccc}
x_{11}+\ldots+x_{1 n} & x_{12} & \ldots & x_{1 n} \\
x_{21} & x_{21}+\ldots+x_{2 n} & \ldots & x_{2 n} \\
\vdots & & \ddots & \vdots \\
x_{n 1} & x_{n 2} & \ldots & x_{n 1}+\ldots+x_{n n}
\end{array}\right)
$$

We do so because under the substitution

$$
\varphi\left(x_{i j}\right)=\left\{\begin{array}{l}
a_{i j} \text { if } i \neq j \\
2 a_{i j} \text { if } i=j,
\end{array}\right.
$$

the matrix $Y$ becomes the matrix $I+A+Q$ we are interested in.

The determinant of $Y$ can be expanded as a polynomial in the variables $x_{i j}$ and analyzed as follows.

$$
\operatorname{det}(Y)=\sum_{J=\left(j_{1} \ldots j_{n}\right) \in I_{n}^{n}}\left(w_{J}\right) P_{J},
$$

where $I_{n}^{n}$ denotes all ordered n-tuples of the integers $1, \ldots n, P_{J}$ denotes the product $x_{1, j_{1}} \ldots x_{n, j_{n}}$ and $w_{J}$ is an integer coefficient dependent on $J$. We will show that under the map $\varphi$, all the terms $w_{J} P_{J}$ are non-negative, and that for a nonbipartite graph there is at least one nonzero term, which will suffice to show that $\operatorname{det}(I+A+Q)>0$. The essential thing now is to study the coefficients $w_{J}$. To this end, we introduce the following definition. 
Definition. Let $S_{J}$ denote the set of all $\sigma \in S_{n}$ such that $P_{J}$ appears in the expansion of $y_{1, \sigma(1)} \ldots y_{n, \sigma(n)}$.

This is a useful definition because of the following equation.

$$
w_{J}=\sum_{\sigma \in S_{J}} \operatorname{sgn}(\sigma) .
$$

A natural way to analyze the set $S_{J}$ is through an auxilliary digraph $D_{J}$. These digraphs $D_{J}$ will allow us to relate the determinant of $I+A+Q$ to the graph $G$ itself. We associate to each ordered n-tuple $J=\left(j_{1} \ldots j_{n}\right)$ a digraph $D_{J}$ on the vertices of $G$ and a diedge from each vertex $i$ to $j_{i}$.

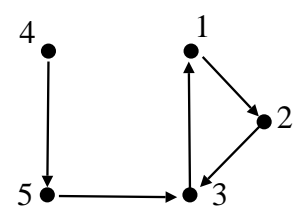

Figure 1: The digraph $D_{J}$ for $J=(23153)$.

To each cycle of the digraph $i_{1} \rightarrow \ldots \rightarrow i_{r}$, we naturally associate the cyclic permutation $\left(i_{1} \ldots i_{r}\right)$ - and denote the set of all such associated cyclic permutations by $C_{J}$. Then we relate the set $S_{J}$ to the set $C_{J}$. First, we need the following lemma.

Lemma 8. The permutations in $C_{J}$ are disjoint.

Proof. Assume there are two nondisjoint cycles $c_{1}$ and $c_{2}$ in $D_{J}$. Then they share some diedge $d=(i \rightarrow j)$. However $D_{J}$ has only one diedge emanating from each vertex, so there is a unique path which begins at the vertex $i$. Since $c_{1}$ and $c_{2}$ are both cycles containing the diedge $d$, we can consider them both as paths begining at the vertex $i$, and thus conclude $c_{2}=c_{1}$.

Let $A_{J}$ denote the set of all permutations $\sigma \in S_{n}$ such that $\sigma$ is a product of a subset of cyclic permutations in $C_{J}$. By the above lemma, $A_{J}$ is equal to the power set of $C_{J}$. Now we are ready to understand the structure of $S_{J}$ through the digraph $D_{J}$.

Lemma 9. For any $J, S_{J}=A_{J}$.

Proof. $S_{J}$ is contained in $A_{J}$.

Take any $\sigma \in S_{J}$. We will show for the cycle decomposition $\sigma=c_{1} \ldots c_{r}$, each $c_{i}$ is in $C_{J}$. Take any such $c_{i}=\left(i_{1} \ldots i_{r}\right)$. Then

$$
\begin{aligned}
y_{1, \sigma(1)} \ldots y_{n, \sigma(n)} & =y_{i_{1}, i_{2} \ldots} \ldots y_{i_{r}, i_{1}} y_{i_{r+1} \sigma\left(i_{r+1}\right)} \ldots y_{i_{n} \sigma\left(i_{n}\right)} \\
& =x_{i_{1}, i_{2}} \ldots x_{i_{r}, i_{1}} y_{i_{r+1} \sigma\left(i_{r+1}\right)} \ldots y_{i_{n} \sigma\left(i_{n}\right)},
\end{aligned}
$$

where the last equality holds because $y_{i_{k}, i_{k+1}}$ are off diagonal terms of $Y$ and hence equal to $x_{i_{k}, i_{k+1}}$. 
By definition, $P_{J}$ appears in the expansion of $y_{1, \sigma(1)} \ldots y_{n, \sigma(n)}$. Hence $x_{i_{1}, i_{2}} \ldots x_{i_{r}, i_{1}}$ is a subfactor of $P_{J}$ and $c_{i}=\left(i_{1} \ldots i_{r}\right)$ is a cycle in $D_{J}$, as desired.

$S_{J}$ contains $A_{J}$.

Take any $\sigma \in A_{J}$. We will show that $P_{J}$ appears as a term in the expansion of $y_{\sigma}=y_{1, \sigma(1)} \ldots y_{n, \sigma(n)}$. Again we decompose $\sigma$ as $\sigma=c_{1} \ldots c_{r}$ where now we assume $c_{i} \in C_{J}$. Consider any factor $x_{k, j_{k}}$ of $P_{J}$.

Case 1. $\sigma$ does not fix $k$.

Then $k$ is permuted by exactly one of the cycles $c_{i}$. Since $x_{k, j_{k}}$ is a subfactor of $P_{J}$, there is a diedge in $D_{J}$ from $k \rightarrow j_{k}$. On the other hand, because $c_{i}$ is in the cycle set of $D_{J}$, there is a diedge in $D_{J}$ from $k \rightarrow c_{i}(k)$. By construction, there is only one diedge in $D_{J}$ from vertex $k$ to any other vertex, and thus $c_{i}(k)$ must equal $j_{k}$.

Returning to $\sigma, y_{\sigma}$ contains the subfactor $y_{k, \sigma(k)}=y_{k, c_{i}(k)}=y_{k, j_{k}}$. Since this is an off diagonal entry, it equals $x_{k, j_{k}}$. So $x_{k, j_{k}}$ appears in every term of the expansion of $y_{\sigma}$.

Case 2. $\sigma(k)=k$.

Then $y_{k, \sigma(k)}=x_{k, 1}+\ldots+x_{k, n}$, so $x_{k, j_{k}}$ arises from this factor.

Therefore, $P_{J}=\prod_{k} x_{k, j_{k}}$ is a term in the expansion of $y_{\sigma}$, and we've shown that $S_{J} \supset A_{J}$.

On the basis of Lemma 9, we can prove the following two propositions.

Proposition 10. If $C_{J}$ contains only odd cycles, then $w_{J}$ is strictly greater than zero.

Proof. Recall that $w_{J}=\sum_{\sigma \in S_{J}} \operatorname{sgn}(\sigma)$. The coefficient $w_{J}$ is positive if there are no even cycles (n-cycle with $\mathrm{n}$ even) in $D_{J}$, because $\operatorname{sgn}(\sigma)$ will be positive for each $\sigma \in S_{J}$. Hence if $C_{J}$ contains only odd cycles, then the sign of any permutation in $S_{J}$ is positive, and $w_{J}$ is positive.

Proposition 11. If $C_{J}$ contains any even cycle, then $w_{J}$ is equal to zero.

However, if there is an even cycle in $D_{J}$, then $w_{J}=0$ as there are the same number of permutations in $S_{J}$ of each sign. Hence

Proof. Let $Q_{J}$ be the set of permutations whose cyclic decomposition consists exclusively of odd cycles in $C_{J}$, and $R_{J}$ be the set of permutations whose cyclic decomposition consists exclusively of even cycles in $C_{J}$.

Then

$$
w_{J}=\sum_{\sigma \in S_{J}} \operatorname{sgn}(\sigma)=\sum_{q \in Q_{J}} \sum_{r \in R_{J}} \operatorname{sgn}(q r)=\sum_{q \in Q_{J}} \sum_{r \in R_{J}} \operatorname{sgn}(r) .
$$

where the last equality holds because $\operatorname{sgn}(q r)=\operatorname{sgn}(q) \operatorname{sgn}(r)$ (as sgn is a homomorphism from $S_{n}$ to $\left.\mathbb{Z} / 2\right)$ and $\operatorname{sgn}(q)$ is guaranteed to be 1 .

Consider just the inner sum. Suppose there are $n$ even cycles in $C_{J}$. Then there are $\left(\begin{array}{l}n \\ k\end{array}\right)$ products of $k$ of these cycles, and each of these products of $k$ cycles has sign $(-1)^{k}$. Thus

$$
\sum_{r \in R_{J}} \operatorname{sgn}(r)=\sum_{k=0}^{n}(-1)^{k}\left(\begin{array}{l}
n \\
k
\end{array}\right)=(1-1)^{n}=0
$$


where the second to last equality can be seen by taking the binomial expansion of $(1+x)^{n}$, and setting $x=-1$. Therefore,

$$
w_{J}=\sum_{q \in Q_{J}} \sum_{r \in R_{J}} \operatorname{sgn}(q r)=0 .
$$

Meanwhile, the map $\varphi$ from the indeterminates $x_{i j}$ to the adjacency matrix entries $a_{i j}$ defined in equation (6) sends $Y$ to $I+A+Q$, and gives that $\operatorname{det}(I+A+Q)=\sum_{J}\left(w_{J}\right) \varphi\left(P_{J}\right)$. Since $w_{J}$ and $\varphi\left(P_{J}\right)$ are both nonnegative integers, $\operatorname{det}(I+A+Q)$ is positive if and only if for some $\mathrm{J}$, both $w_{J}$ and $\varphi\left(P_{J}\right)$ are nonzero.

The key reason for introducing the digraphs $D_{J}$ is now apparent - $P_{J}$ is nonzero if and only if $D_{J}$ is inscribed in $G$, and $w_{J}$ is nonzero if and only if $D_{J}$ contains no even cycle. Hence the product $w_{J} P_{J}$ is nonzero for some $J$ if and only if a primitive digraph with no even cycles can be inscribed in $G$. The fact that this can be done when $G$ is nonbipartite is the content of Lemma 12.

Lemma 12. If $G$ is a connected nonbipartite graph, there exists a primitive digraph of $G$ which contains exactly one odd cycle.

Proof. By assumption, $G$ contains some cycle $C$ of odd length. Take such a cycle and remove an edge to obtain an acyclic subgraph of $G$. Extend this acyclic subgraph of $G$ to a spanning tree of $G$, then add back the removed edge of the cycle $C$ to obtain a subgraph $H$ of $G$. We can obtain a primitive digraph from $H$ by giving each edge of $H$ a direction in such a way that each vertex of $G$ has exactly one diedge pointing from it.

First, consider the subgraph $F$ of $H$ obtained by removing the edges in the cycle $C$. This leaves a spanning forest of $G$, (allowing single vertices to be counted as trees). Each of the trees contains exactly one vertex of the cycle $C$ - label this vertex as the root, and to every other vertex of the tree, assign it the diedge pointing to its parent vertex.

We have now assigned one diedge to each vertex in $H$ except to those vertices in $C$. For these, simply pick a direction to traverse the cycle in, and assign to each vertex the diedge pointing to the next vertex in the cycle. Now we have a primitive digraph with exactly one odd cycle.

Thus we have seen that

$$
\operatorname{det}(I+A+Q)=\varphi(\operatorname{det}(Y))=\sum_{J} w_{J} \varphi\left(P_{J}\right)
$$

that the last expression is a sum of nonnegative terms, and finally, that if $G$ is connected there is a strictly positive term in the sum if and only if $G$ is not bipartite, yielding the desired result.

\section{$5 \quad$ IV. Degenerate Families}

We have seen that for connected md2 graphs, many invariants of the graph can be computed from the zeta function. Is the graph itself then determined? The answer is no, 
as the following infinite family of pairs of connected but non-isomorphic graphs with the same zeta function shows.

For any two nonnegative integers $l, m$, let $F R_{l, m}$ and $F L_{l, m}$, be:
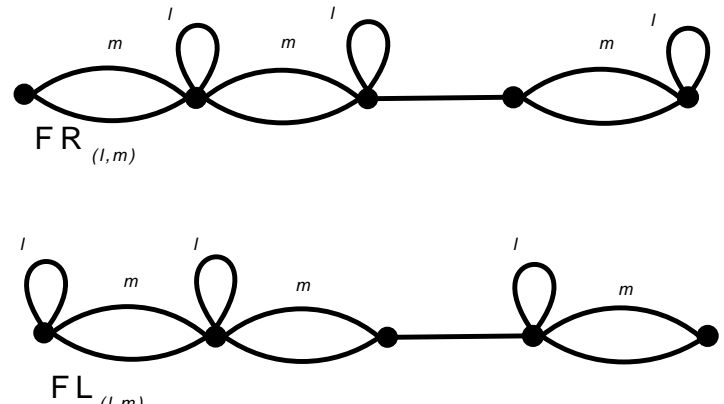

where $l$ is the number of loops on each vertex, and $m$ is the number of extra edges between the designated vertices. For example, the above graphs are $F R_{1,1}$ and $F L_{1,1}$, the original pair discovered by Czarneski.

Theorem 13. The graphs $F R_{l, m}$ and $F L_{l, m}$ have the same zeta function.

Proof. The pair were shown to have the same zeta function by evaluating Bass' formula (2) for each graph with Mathematica, and showing that the resulting functions are equal.

A similar family is the following. For any four nonnegative integers $k, l, m, n$, let $S R_{k, l, m, n}$ and $S L_{k, l, m, n}$ be:

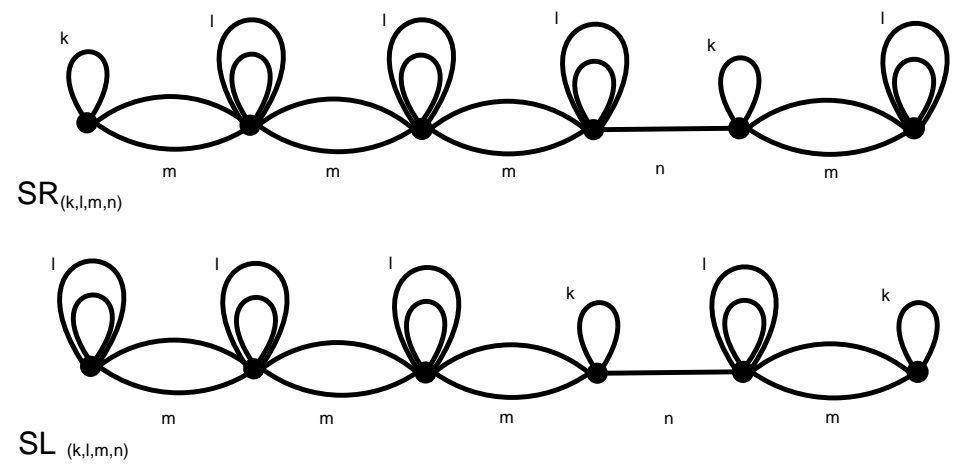

where again, $k$ and $l$ are the number of loops on the indicated vertices, and $m$ and $n$ are the number of extra edges between the indicated vertices. For example, the two graphs above depict $S R_{1,2,1,0}$ and $S L_{1,2,1,0}$.

Theorem 14. The graphs $S R_{k, l, m, n}$ and $S L_{k, l, m, n}$ have the same zeta function in the following cases:

1. $k=0, n=0$, and arbitrary natural numbers $m, l$.

2. $m=\frac{(a-1)(b-1)}{2}, l=\frac{a+b}{2}$, for some odd natural numbers $a, b$ and arbitrary $n, k$. 
Proof. Case 1 concerns a family very similar to the pairs $F R_{l, m}$ and $F L_{l, m}$ discussed above, and a direct calculation in mathematica shows the two graphs have equal zeta functions.

In Case 2, again a direct check using mathematica confirms that the two graphs have equal zeta functions. To find the given conditions on $m$ and $l$, the zeta functions for $S R_{k, l, m, n}$ and $S L_{k, l, m, n}$ were computed with $k, l, m, n$ arbitrary, and then setting the functions equal one can solve for the conditions on $m$ and $l$.

The nature of these restrictions on $k, l, m$ and $n$ may give clues for further research as to what is and is not determined by the zeta function for connected graphs. Additionally, thus far we have obtained infinite families of pairs of graphs with the same zeta function by adding edges, but one might consider extending the family by adding vertices.

Conjecture 1. An infinite family of pairs of graphs with the same zeta function can be obtained from $F R_{(1,1)}$ and $F L_{(1,1)}$ by iterating the following process: connect the leftmost vertex $\ell$ to a new vertex $n$ with two edges, and on the first graph add a loop on $\ell$ while on the second graph, add a loop on $n$.

$S R_{(0,1,1,0)}$ and $S L_{(0,1,1,0)}$ are a first step in this direction, extending vertex number from five to six. Proving this conjecture and exploring which degrees of freedom such as $k, l, m, n$ can be introduced as well as the relations forced between them may give useful insight into what the zeta function does determine for connected graphs. Computations up to 12 vertices have shown that these graphs have the same zeta function.

Czarneski also gave an example of a pair of graphs with the same zeta function but differing numbers of vertices and components. Here we extend that example to an infinite family. For any odd natural number $r$, let $P_{r}$ denote the graph on one vertex with $\frac{r+1}{2}$ loops. For any natural numbers $m, n$, let $M_{(m, n)}$ be the graph on two vertices with $m$ edges between the two vertices, and $n$ loops on each vertex.
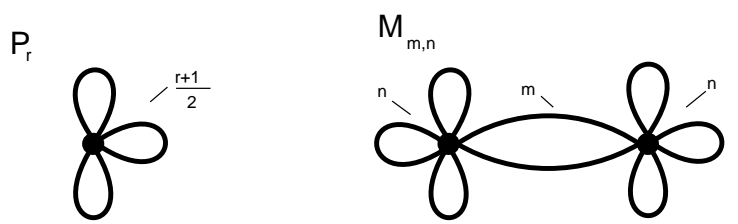

For each pair of odd integers $a, b \geq 3$, the following graphs $\Phi_{(a, b)}$ and $\Psi_{(a, b)}$ have the same zeta function $Z_{\Phi_{(a, b)}}(u)=Z_{\Psi_{(a, b)}}(u)=\frac{1}{\left(1-u^{2}\right)^{a b-1}(u-1)^{3}(a b u-1)(a u-1)(b u-1)}$.

$$
\begin{array}{|l|}
\hline \Phi_{(a, b)}=M_{\left(\frac{(a-1)(b-1)}{2}, \frac{(a+1)(b+1)}{4}\right)} \coprod P_{1} \\
\hline \Psi_{(a, b)}=\left(\frac{(a-1)(b-1)}{4} \times M_{2,0}\right) \coprod P_{a b} \coprod P_{a} \coprod P_{b} . \\
\hline
\end{array}
$$

Czarneski's original example was the smallest pair in this family, $\Phi_{(3,3)}, \Psi_{(3,3)}$. This family of nonconnected graphs shows that connectedness is an essential hypothesis for the result that the vertex number of $G$ can be computed from $Z_{G}(u)$. We can see how the zeta function fails to capture the vertex number. Recall Theorem 7 stated that $1-u^{2}$ 
divides $\operatorname{det}\left(I-A u+Q u^{2}\right)$ once for each bipartite noncyclic component, and twice for each n-cycle where $\mathrm{n}$ is even. In this example, $\Phi_{a, b}$ and $\Psi_{a, b}$ are both nonbipartite, but have different numbers of bipartite components of the two types, which is why they are able to have the same zeta function but different component numbers and values for $n-e$.

In fact, this family shows that even $c-n$, where $c$ is the number of components and $n$ the number of vertices, is not preserved by the zeta function.

\section{The $\mathrm{T}$ matrix}

As noted in the introduction, for an md2 graph $G, Z_{G}(u)$ is closely related to the spectrum of the T matrix. Thus recovering $G$ from the $\mathrm{T}$ matrix is related to recovering $G$ from $Z_{G}(u)$. The first step toward proving Theorem 4 is the following lemma, which recovers $G$ locally from $\mathrm{T}$.

Lemma 15. If a diedge $d$ points to $\geq 2$ diedges, then $\bar{d}$ can be determined from $T$.

Proof. Take a diedge $a$ which $d$ points to. By hypothesis $d$ points to $\geq 2$ diedges, so there are $\geq 2$ diedges which point to $a$. Specifically, there is a second diedge $b$ which also points to $a$. Furthermore, the only diedge which $b$ points to that $d$ does not is $\bar{d}$. By comparing $T(b)$ to $T(d), \bar{d}$ can be determined.

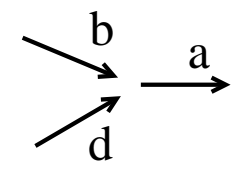

A similar argument shows that if $d$ is pointed to by $\geq 2$ diedges, then $\bar{d}$ can be determined from $T$. Let $A$ denote the set of diedges $d$ in $\vec{G}$ such that $d$ points to two or more diedges, or is pointed to by two or more diedges. For all diedges $d$ in $A$, we have seen that we can recover $\bar{d}$, and we will see below that this allows us to reconstruct the graph near these diedges.

To recover the rest of $G$, we introduce the concept of a 2-path.

Definition. A 2-path is a sequence of diedges $d_{1} \ldots d_{r}$ such that for each $i, T\left(d_{i}\right)=d_{i+1}$.

Note that a 2-path is defined only for diedges which point to or are pointed to by a single other diedge. Let $B$ denote the set of diedges $d$ in $\vec{G}$ such that the maximal 2-path $d_{1} \rightarrow \ldots \rightarrow d_{r}$ containing $d$ terminates at least on one side in a vertex of degree $\geq 3$. Let $C$ denote the remaining diedges in $\vec{G}$ not in either $A$ or $B$ - for $d \in C$, either the maximal 2-path containing $d$ is cyclic and $T\left(d_{r}\right)=d_{1}$, or the maximal 2-path containing $d$ terminates in each direction.

No diedge in $A \cup B$ is connected to any in $C$, so any component of $\vec{G}$ contains diedges entirely from $A \cup B$ or entirely from $C$. Let $G_{A \cup B}$ denote the components of $G$ which gave rise to components of $\vec{G}$ which contain only diedges from $A \cup B$. Let $G_{C}$ be analogous. Clearly if $G$ has no isolated vertices, $G=G_{A \cup B} \cup G_{C}$. 
Lemma 16. From the $T$ matrix, $G_{A \cup B}$ can be reconstructed.

Proof. For any diedge $d_{i}$ in $B, \bar{d}_{i}$ can be determined from $T$. Specifically, let $d_{1} \ldots d_{r}$ be the maximal 2-path containing $d_{i}$. Either $d_{1}$ is pointed to by $\geq 2$ diedges, and $\bar{d}_{1}$ can be determined by lemma 15 , or $d_{r}$ points to $\geq 2$ diedges, meaning that $\bar{d}_{r}$ can be determined. In the former case, $T^{i-1}\left(\bar{d}_{1}\right)=\bar{d}_{i}$, and the latter case is similar. We saw above that for any diedge $d$ in $A, \bar{d}$ could be recovered.

Now we recover the vertex set of $G_{A \cup B}$ by introducing the following partition of $A \cup B$ : for each diedge $d_{i}, d_{i}$ together with all diedges which point to $\bar{d}_{i}$ form a class - and we consider this class to be a vertex. The reader can check that this gives a well defined partition of the set $A \bigcup B$. Clearly, two vertices $\left[d_{i}\right]$ and $\left[d_{j}\right]$ are adjacent if and only if there are representatives $e_{i}$ of the class $\left[d_{i}\right]$ and $e_{j}$ of the class $\left[d_{j}\right]$ such that $\bar{e}_{i}=e_{j}$. Hence we have recovered the vertex adjacency matrix of $G_{A \cup B}$, and thus $G_{A \cup B}$ itself.

Lemma 17. From the T matrix, $G_{C}$ can be reconstructed.

Proof. Consider the set $M$ of all maximal 2-paths in $C$. Each maximal 2-path will be either a cycle, or a line that terminates on each end. Thus $M$ will be a set of cycles and lines.

Now, if we start with an n-cycle and replace each edge by one diedge in each direction to get a directed graph, we will obtain two disjoint directed n-cycles. Similarly for lines of length n. Hence, for any natural number $n, G_{C}$ will contain exactly half as many n-cycles and half as many n-lines as there are in $M$.

Hence, we have reconstructed $G_{C}$.

These results prove Theorem 4 .

Proof of Theorem 4. Because $G$ has no isolated vertices, $G=G_{A \cup B} \cup G_{C}$. Hence by Lemmas 16 and 17, $G$ can be reconstructed from $T$.

\section{References}

[1] H. Bass. The Ihara-Selberg zeta function of a tree lattice. Int. J. Math., 3:717-797, 1992.

[2] D. Czarneski. Zeta functions of finite graphs. PhD thesis, Louisiana State University, 2005.

[3] K. Hashimoto. Zeta functions of finite graphs and representations of p-adic groups. Stud. Pure Math., 15:211-280, 1989.

[4] Y. Ihara. On discrete subgroups of the two by two projective linear group over p-adic fields. J. Math. Soc. Japan, 18:219-235, 1966.

[5] A. Mellein. Zeta functions and spectra of regular md2 graphs. Not published, 2001. 